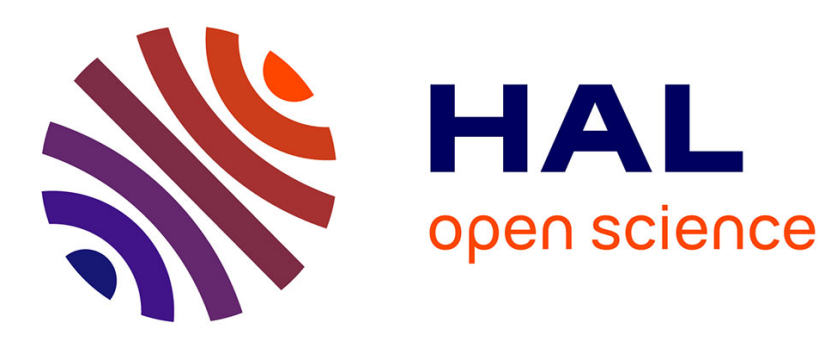

\title{
On the Steady Solution of Non-Linear Advection equations in Steady Recirculating Flows
}

\author{
Guillaume Chaidron, Francisco Chinesta
}

\section{To cite this version:}

Guillaume Chaidron, Francisco Chinesta. On the Steady Solution of Non-Linear Advection equations in Steady Recirculating Flows. Computer Methods in Applied Mechanics and Engineering, 2002, 191 (11-12), pp.1159-1172. 10.1016/S0045-7825(01)00319-X . hal-00020721

\section{HAL Id: hal-00020721 \\ https://hal.science/hal-00020721}

Submitted on 15 Nov 2017

HAL is a multi-disciplinary open access archive for the deposit and dissemination of scientific research documents, whether they are published or not. The documents may come from teaching and research institutions in France or abroad, or from public or private research centers.
L'archive ouverte pluridisciplinaire HAL, est destinée au dépôt et à la diffusion de documents scientifiques de niveau recherche, publiés ou non, émanant des établissements d'enseignement et de recherche français ou étrangers, des laboratoires publics ou privés. 


\title{
On the steady solution of non-linear advection equations in steady recirculating flows
}

\author{
G. Chaidron *, F. Chinesta \\ Laboratoire de Rhéologie et Thermodynamique des Matériaux Macromoléculaires, CNAM, \\ Conservatoire National des Arts et Mee'tiers, 292 rue Saint Martin, 75141 Paris Cedex 03, France
}

\begin{abstract}
Many physical problems like short fiber suspensions flows or viscoelastic flows are modeled by linear and non-linear advection equations. Many of the experimental and industrial flows show often steady recirculating areas which introduce some additional difficulties in the numerical simulation. Actually, the advection equation is supposed to have a steady solution in these steady recirculating flows but neither boundary conditions nor initial conditions are known in such flows. In this paper, we present accurate techniques to solve non-linear advection equations defined in steady recirculating flows. These techniques combine a standard treatment of the non-linearity with a more original treatment of the associated linear problems.
\end{abstract}

Keywords: Non-linear advection equation; Steady recirculating flows; Non-Newtonian fluid mechanics

\section{Introduction}

Numerical modeling of non-Newtonian fluid flows involves usually the coupling between motion equations, which lead to an elliptic problem, and the fluid constitutive equation, which introduces an advection problem related to the fluid history.

For example, for short fiber reinforced thermoplastic (SFRT) flow models, the extra-stress tensor $\underline{\underline{\tau}}$ depends on the fiber orientation [1-8]

$$
\underline{\underline{\tau}}=2 \mu\left\{\underline{\underline{D}}+N_{\mathrm{p}} \operatorname{Tr}(\underline{\underline{s}} \underline{\underline{D}}) \underline{\underline{S}}\right\}
$$

where $\mu$ is the equivalent suspension viscosity, $N_{\mathrm{p}}$ a scalar parameter depending on both the fiber concentration and the fiber aspect ratio, $\underline{\underline{D}}$ the strain rate tensor, $\operatorname{Tr}()$ denotes the tensorial operator trace and $\underline{\underline{S}}$ is the second-order orientation tensor defined by

$$
\underline{\underline{s}}=\frac{\underline{\underline{a}}}{\operatorname{Tr}(\underline{\underline{a}})} .
$$

From a physical point of view, we can consider that the eigenvalues of the orientation tensor $\underline{\underline{s}}$ represent the probability of finding the fiber in the direction of the corresponding eigenvectors.

\footnotetext{
${ }^{*}$ Corresponding author. Tel.: +33-1-40272401; fax: +33-1-40272526.

E-mail address: chaidron@cnam.fr (G. Chaidron).
} 
The evolution of the tensor $\underline{\underline{a}}$ is given by the advection equation

$$
\frac{\mathrm{d} \underline{\underline{a}}}{\mathrm{~d} t}=\underline{\underline{\Omega}} \underline{\underline{a}}-\underline{\underline{a}} \underline{\underline{\Omega}}+k(\underline{\underline{D}} \underline{\underline{a}}+\underline{\underline{a}} \underline{\underline{D}})+D_{r}\left(\underline{\underline{a}}-\frac{\underline{\underline{I}}}{3}\right)
$$

where $\underline{\underline{\Omega}}$ represents the vorticity tensor, $k$ is a constant which depends on the fibers aspect ratio, and $D_{r}$ is a diffusion coefficient.

Other examples are the viscoelastic models. In most of the differential viscoelastic models [9,10], the evolution of the extra-stress tensor $\underline{\underline{\tau}}$ is given by an advection equation. For example for the viscoelastic Maxwell's model we have

$$
\lambda \frac{\delta \underline{\underline{\underline{\tau}}}}{\delta t}+\underline{\underline{\tau}}=2 \mu \underline{\underline{D}}
$$

where $\delta / \delta t$ denotes the convected derivative

$$
\frac{\delta \underline{\underline{\tau}}}{\delta t}=\frac{\mathrm{d} \underline{\underline{\tau}}}{\mathrm{d} t}-\operatorname{Grad} \underline{v} \underline{\underline{\tau}}-\underline{\underline{\tau}}(\operatorname{Grad} \underline{v})^{\mathrm{T}}
$$

and $\lambda$ is a relaxation time.

In the recent Pom-Pom viscoelastic model, as described in [11], the extra-stress tensor depends on both the molecular orientation $\underline{\underline{s}}$ and the molecular extension $\lambda$

$$
\underline{\underline{\tau}}=\alpha \lambda^{2} \underline{\underline{s}},
$$

where $\alpha$ is a material parameter.

Both evolutions are also given by two advection equations

$$
\begin{aligned}
& \frac{\mathrm{d} \underline{\underline{a}}}{\mathrm{~d} t}=\underline{\underline{\Omega}} \underline{\underline{a}}-\underline{\underline{a}} \underline{\underline{\Omega}}+\underline{\underline{D}} \underline{\underline{a}}+\underline{\underline{a}} \underline{\underline{D}}-\frac{1}{\tau_{b}}\left(\underline{\underline{a}}-\frac{\underline{\underline{I}}}{3}\right), \\
& \underline{\underline{s}}=\frac{\underline{\underline{a}}}{\operatorname{Tr}(\underline{\underline{a}})}
\end{aligned}
$$

and

$$
\frac{\mathrm{d} \lambda}{\mathrm{d} t}=\lambda \operatorname{Tr}(\underline{\underline{s}} \underline{\underline{D}})-\frac{1}{\tau_{s}}(\lambda-1),
$$

where $\tau_{b}$ and $\tau_{s}$ are two relaxation times.

The molecular orientation equation may be written in the form

$$
\frac{\mathrm{d} \underline{\underline{s}}}{\mathrm{~d} t}=\underline{\underline{\Omega}} \underline{\underline{s}}-\underline{\underline{s}} \underline{\underline{\Omega}}+\underline{\underline{D}} \underline{\underline{s}}+\underline{\underline{s}} \underline{\underline{D}}-2 \operatorname{Tr}(\underline{\underline{s}} \underline{\underline{D}}) \underline{\underline{s}}-\frac{1}{\tau_{b}^{*}}\left(\underline{\underline{s}}-\frac{\underline{\underline{I}}}{3}\right)
$$

where $\tau_{b}^{*}=\tau_{b} \operatorname{Tr}(\underline{a})=\tau_{b} \lambda^{2}[12]$.

Thus, generally, such a model is defined from the equations of motion where the mass and inertia terms may be neglected, and from the extra-stress tensor evolution.

The equations of motion define an elliptic problem and the extra-stress tensor evolution equation defines a hyperbolic problem.

The discretization of an advection equation is a difficult matter. It can be carried out integrating its Lagrangian description by means of the method of characteristics [13-18], or by using the Eulerian discretization of its variational formulation: Streamline Upwind (SU) or Streamline Upwind Petrov-Galerkin (SUPG) finite elements, [7,9,10,19-22]; discontinuous finite elements [10,23]; or discontinuous finite volumes [22]. In the case of a SFRT flow or a Pom-Pom viscoelastic model among others, the interpolation of the orientation tensors (required if standard Eulerian discretization techniques are used) introduces nonphysical orientation effects [18,22]. 
Such numerical models are made in order to simulate real industrial processes and to predict resulting mechanical properties of conformed pieces afterwards. Many of the experimental and industrial flows show recirculating areas $[24,25]$. For example, in a lid-driven cavity problem, the flow recirculates under the influence of a moving plate. In a contraction or in an expansion flow (as encountered, for example, in extrusion processes), various recirculating areas are observed. Most of these phenomena are associated with a steady state of the flow, which introduces some additional difficulties in the numerical simulation. Actually, the advection equation is supposed to have a steady solution in these steady recirculating flows but neither boundary conditions nor initial conditions are known in such flows [18].

In a former paper [26] we have proved that linear advection problems in steady recirculating flows have usually only one steady solution for general flow kinematics. We also give in [26] a numerical procedure to determine this steady solution by imposing its periodicity along the closed trajectories required in steady regimes. This accurate solution may be used in the context of steady simulations to obtain the solution in the steady recirculating parts of the flow as well as to check the accuracy of other discretization techniques. In this work this former strategy is generalized for the treatment of non-linear models as encountered usually in the non-Newtonian fluid mechanics. Non-linear problems are transformed in a sequence of linear ones with an iterative procedure. Each linear problem is then solved keeping the periodicity condition along the closed streamlines.

\section{Related works}

\subsection{Lagrangian strategies}

In [27], a first Lagrangian strategy to solve the non-linear advection equation related to the fiber orientation in steady recirculating flows of short fibers suspensions was presented. In order to summarize this technique we will consider the non-linear scalar advection equation defined by

$$
\frac{\mathrm{d} a}{\mathrm{~d} t}+f(a)=g,
$$

where $f(a)$ represents the non-linear term, $g$ is a function depending only on spatial coordinates, and $\mathrm{d} a / \mathrm{d} t$ denotes the material derivative

$$
\frac{\mathrm{d} a}{\mathrm{~d} t}=\frac{\partial a}{\partial t}+\underline{v} \cdot \operatorname{Grad} a .
$$

We assume that Eq. (11) is defined in a steady recirculating flow. In this case Eq. (11) results ill-defined because neither initial conditions nor boundary conditions are known along the closed trajectories.

The numerical strategy proposed consists in integrating the non-linear equation by means of the method of characteristic from an arbitrary initial value $a^{0}$ in a point $P$ on the closed trajectory as depicted in Fig. 1. After one rotation, the initial point is reached, and from the integration of Eq. (11) the value of $a$ at point $P$ will be $a(T)$, which in general is different of $a^{0}$. In order to impose the periodicity of the steady solution, a correction of the initial value $a^{0}$ is needed. If we use a Newton's method to calculate this initial value correction, we need to define the residual associated with $a^{0}, R\left(a^{0}\right)$

$$
R\left(a^{0}\right)=a(T)-a^{0} .
$$

Let now $\Delta a$ be the correction of $a^{0}$ in order to obtain a zero residual

$$
R\left(a^{0}+\Delta a\right)=0=R\left(a^{0}\right)+\left.\frac{\partial R}{\partial a}\right|_{a=a^{0}} \Delta a+\Theta\left(\Delta a^{2}\right)
$$

from which the linearized correction $\overline{\Delta a}$ results

$$
\overline{\Delta a}=-\frac{R\left(a^{0}\right)}{\left.\frac{\partial R}{\partial a}\right|_{a=a^{0}}} .
$$




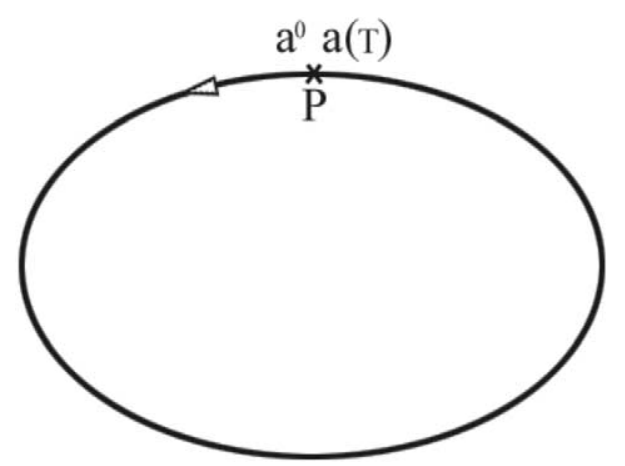

Fig. 1. Looking for a periodic solution.

In order to evaluate numerically the term $\left.\left(\frac{\partial R}{\partial a}\right)\right|_{a=a^{0}}$, we integrate again from another initial value at the same point $P$, given by $a^{0}+\epsilon(\epsilon$ is representing a small value). After a turn along the closed trajectory, the new value of $a$ associated with the initial value $a^{0}+\epsilon$ will be $a^{\epsilon}(T)$

$$
\left.\frac{\partial R}{\partial a}\right|_{a=a^{0}}=\frac{R\left(a^{0}+\epsilon\right)-R\left(a^{0}\right)}{\epsilon}=\frac{\left(a^{\epsilon}(T)-\left(a^{0}+\epsilon\right)\right)-\left(a(T)-a^{0}\right)}{\epsilon} .
$$

This procedure must be continued until reaching convergence, i.e. $|\overline{\Delta a}|<\delta$ (where $\delta$ is another parameter small enough).

Another strategy very similar to the previous one consists in integrating the non-linear advection equation around the streamline until reaching a steady solution [27]. It is easy to verify that this technique can be considered as equivalent to the previous one when the initial value correction is searched by means of a fixed point strategy

$$
\Delta a=a(T)-a^{0} .
$$

In these techniques the non-linear equation is solved with accuracy at each iteration, and the solution periodicity is searched by means of an iterative scheme, e.g. Newton's method or fixed point algorithm.

Sometimes, the steady solution can be found by the integration of the evolution equation, using the method of characteristics, until reaching the steady state. This technique is applied in [17] on the simulation of steady recirculating flows of short fibers suspensions. However, as it is shown in [28], the steady solution can only be reached for a particular choice of the initial condition. For other initial conditions, the steady state may not exist.

\subsection{Eulerian strategies}

A second family of techniques consists in integrating the Eulerian formulation of Eq. (11) taking into account its hyperbolic character. Thus, a first strategy consists in solving the evolution problem until reaching the steady state regime from an arbitrary initial condition $a(t=0)$. In [29], an Eulerian discretization of the fiber orientation evolution problem was considered, using Fourier's polynoms to approximate the orientation tensor on the closed trajectories in order to enforce solution periodicity at each time step.

Another possibility consists in searching directly the steady solution using a fixed point or a Newton's method combined with a Fourier's interpolation of the solution on the streamlines.

In relation to the first family of techniques (described in the previous section), these Eulerian strategies allows us to keep the solution periodicity. Either the steady solution of the problem is obtained by solving the evolution problem until reaching the steady state or it is obtained by means of a standard linearization of the non-linear problem. However, so far, techniques keeping the solution periodicity require a periodic interpolation of the unknown field on the closed trajectories (for example using Fourier's polynoms) and the resolution of linear systems, except if we choose an explicit strategy for the temporal discretization of the evolution problem. 
Non-linear advection equations are usually linearized and solved using a well adapted method to its hyperbolic character like SU, SUPG, discontinuous Galerkin or discontinuous finite volume methods,... (see for example [30] and associated references). For example, this strategy was applied to a fiber suspension in an abrupt contraction flow [21]. These techniques give a steady Eulerian solution coming from the iterative procedure used in the non-linearity treatment. However, we can show that the stationarity of the solution is not locally obtained because the periodicity of the solution along the closed streamlines is not verified. For example, starting from the Eulerian solution on a point, if we integrate Eq. (11) along the closed streamline, the solution after one turn is different from the initial one. In this case, we can conclude that the Eulerian solution is not the steady one.

\section{Solution of a non-linear advection problem}

In a former paper [26], we have proved that linear steady advection problems in steady recirculating flows have in general only one steady solution for general flow kinematics. We have also given a numerical procedure for its determination. In the present paper, we generalize this numerical procedure to solve nonlinear advection problems. The proposed technique combines a standard linearization strategy (for example a fixed point or a Newton's technique) with a solution at each iteration of the associated linear problem. This linear problem will be solved keeping the solution periodicity on the closed trajectories, avoiding Eulerian interpolations and linear systems solutions.

Using any linearization technique, a non-linear problem can be transformed in a sequence of linear problems. In order to keep the solution periodicity of each linear problem, we use the technique described in [26] and summarized below.

\subsection{Steady solution of linear advection equations in steady recirculating flows}

In order to offer more generality to the following discussion we will consider at this point and according to [26] a general system of linear advection equations

$$
\frac{\mathrm{d} \underline{a}}{\mathrm{~d} t}=\underline{A}(\underline{x}(t)) \underline{a}+\underline{B}(\underline{x}(t)),
$$

where the matrix $\underline{A}$ and the vector $\underline{B}$ depend only on the flow kinematics, and thus on the particle position given by the integration of

$$
\frac{\mathrm{d} \underline{x}}{\mathrm{~d} t}=\underline{v}(\underline{x}(t)) .
$$

Without any loss in generality, we consider the unknown vector $\underline{a}$ composed by three scalar components

$$
\underline{a}=\left(\begin{array}{l}
a_{1} \\
a_{2} \\
a_{3}
\end{array}\right) .
$$

The differential system (17) is linear so that its general solution can be written as the sum of the general solution of the homogeneous differential system

$$
\frac{\mathrm{d} \underline{a}}{\mathrm{~d} t}=\underline{\underline{A}}(\underline{x}(t)) \underline{a}
$$

and a particular solution of the non-homogeneous differential system.

It is well known that for a linear homogeneous differential system, the linear combination of solutions is also a solution of the differential system. Thus if we denote by $\underline{a}_{h}^{(1)}(t), \underline{a}_{h}^{(2)}(t)$ and $\underline{a}_{h}^{(3)}(t)$, the solutions of the differential system (20) associated with the initial conditions 


$$
\begin{aligned}
& \underline{a}_{h}^{(1)}(t=0)=\left(\begin{array}{l}
1 \\
0 \\
0
\end{array}\right), \\
& \underline{a}_{h}^{(2)}(t=0)=\left(\begin{array}{l}
0 \\
1 \\
0
\end{array}\right),
\end{aligned}
$$

and

$$
\underline{a}_{h}^{(3)}(t=0)=\left(\begin{array}{l}
0 \\
0 \\
1
\end{array}\right),
$$

respectively, then the solution of Eq. (20) associated with the initial condition

$$
\underline{a}_{h}(t=0)=\underline{\alpha}=\left(\begin{array}{l}
\alpha_{1} \\
\alpha_{2} \\
\alpha_{3}
\end{array}\right)
$$

with $\left(\alpha_{1}, \alpha_{2}, \alpha_{3}\right) \in \mathbb{R}^{3}$, will be given by

$$
\underline{a}_{h}(t)=\alpha_{1} \underline{a}_{h}^{(1)}(t)+\alpha_{2} \underline{a}_{h}^{(2)}(t)+\alpha_{3} \underline{\underline{a}}_{h}^{(3)}(t) .
$$

Since we are looking for a particular solution, we proceed by integrating Eq. (17) from an arbitrary initial condition given by

$$
\underline{a}_{c}(t=0)=\left(\begin{array}{l}
\beta_{1} \\
\beta_{2} \\
\beta_{3}
\end{array}\right)
$$

from which the particular solution $\underline{a}_{c}(t)$ is obtained.

The general solution of the system (17), $\underline{a}(t)$, is then

$$
\underline{a}(t)=\alpha_{1} \underline{a}_{h}^{(1)}(t)+\alpha_{2} \underline{a}_{h}^{(2)}(t)+\alpha_{3} \underline{a}_{h}^{(3)}(t)+\underline{a}_{c}(t)
$$

and its initial value is

$$
\underline{a}(t=0)=\left(\begin{array}{c}
\alpha_{1}+\beta_{1} \\
\alpha_{2}+\beta_{2} \\
\alpha_{3}+\beta_{3}
\end{array}\right) .
$$

For steady recirculating flows, the only natural condition to impose to the solution, is its periodicity along the closed trajectories [18]. Thus, the solution after a rotation of period $T$ must be the initial value

$$
\underline{a}(t=0)=\alpha_{1} \underline{a}_{h}^{(1)}(T)+\alpha_{2} \underline{a}_{h}^{(2)}(T)+\alpha_{3} \underline{a}_{h}^{(3)}(T)+\underline{a}_{c}(T)
$$

this defines the linear system

$$
\underline{\underline{C}} \underline{\alpha}=\underline{D} .
$$

From this system, we can obtain the values of the three components of the vector $\underline{\alpha}$. Thus, the steady solution of the advection equation on the closed streamlines is defined by Eq. (27). This general solution is unique if it exists. If not, the number of solutions is infinite [26]. 


\subsection{Linearization}

Eq. (11) can be linearized using for example a fixed point strategy. Then, this equation is written in the iterative form

$$
\frac{\mathrm{d} a^{n}}{\mathrm{~d} t}+h\left(a^{n-1}\right) a^{n}=g,
$$

where $a^{n}$ denotes the solution at iteration $n$ and the non-linear function $f(a)$ has been decomposed as

$$
f(a)=h(a) a,
$$

where $h(a)$ is eventually another non-linear function. The simplest linearization technique consists in considering the term $h(a)$ at the previous iteration $h\left(a^{n-1}\right)$ as assumed in Eq. (31). The iterative scheme starts from an arbitrary periodic function $a^{0}$. This algorithm allows us to find a solution of the non-linear equation (11) keeping the solution periodicity at each iteration if the technique described in the previous section is used to solve the associated linear problems.

Another usual linearization technique that can be used to solve our problem is Newton's method. In this case, the residual associated with the $(n-1)$ th iteration solution, $a^{n-1}$, of Eq. (11) is defined by

$$
R\left(a^{n-1}\right)=\frac{\mathrm{d} a^{n-1}}{\mathrm{~d} t}+f\left(a^{n-1}\right)-g .
$$

If $R\left(a^{n-1}\right) \neq 0$, then a correction of $a^{n-1}$ denoted by $\Delta a$ is required. Newton's linearization is then

$$
\frac{\mathrm{d} \Delta a}{\mathrm{~d} t}+\left.\frac{\partial f}{\partial a}\right|_{a=a^{n-1}} \Delta a=-R\left(a^{n-1}\right) .
$$

If this linear equation is solved keeping its periodicity along the closed trajectory (as described above), $\Delta a$ will be periodic and in consequence

$$
a^{n}=a^{n-1}+\Delta a
$$

will also be periodic. The iteration algorithm stops when the residual is small enough. Thus, the resulting solution is a steady solution of the original non-linear problem because the associated residual is quasi- zero and is periodic on the closed streamline.

These linearization techniques allow us to find a steady solution of a non-linear advection equation avoiding global Eulerian interpolations along the trajectories and the solution of the associated linear equations systems.

From another point of view, these techniques establish a duality with the strategy described previously and which is looking for a periodic solution keeping the verification of the non-linear equation. In the present case, the periodicity is kept, and the verification of the non-linear equation is searched.

\section{Results and discussion}

To illustrate the computational implementation of the proposed strategy, a lid-driven steady cavity flow is considered. We will focus our discussion on the solution of advection problems governing molecular orientation and molecular extension for the POM-POM viscoelastic model presented in Section 1. In the present study we neglect the effects of the molecular orientation and the molecular extension on the flow kinematics, which in consequence is described by the solution of the Stokes problem defined by

$$
\begin{aligned}
& \nabla p=\mu \Delta \underline{v} \quad \text { in }] 0,1[\times] 0,1[ \\
& \underline{v}=\underline{0} \text { on } x=0, x=1, y=0, \\
& (u, v)=\left(-\frac{2}{5} x(x-1), 0\right) \quad \text { on } y=1,
\end{aligned}
$$

where $u$ and $v$ are the components of the velocity vector $\underline{v}$. 


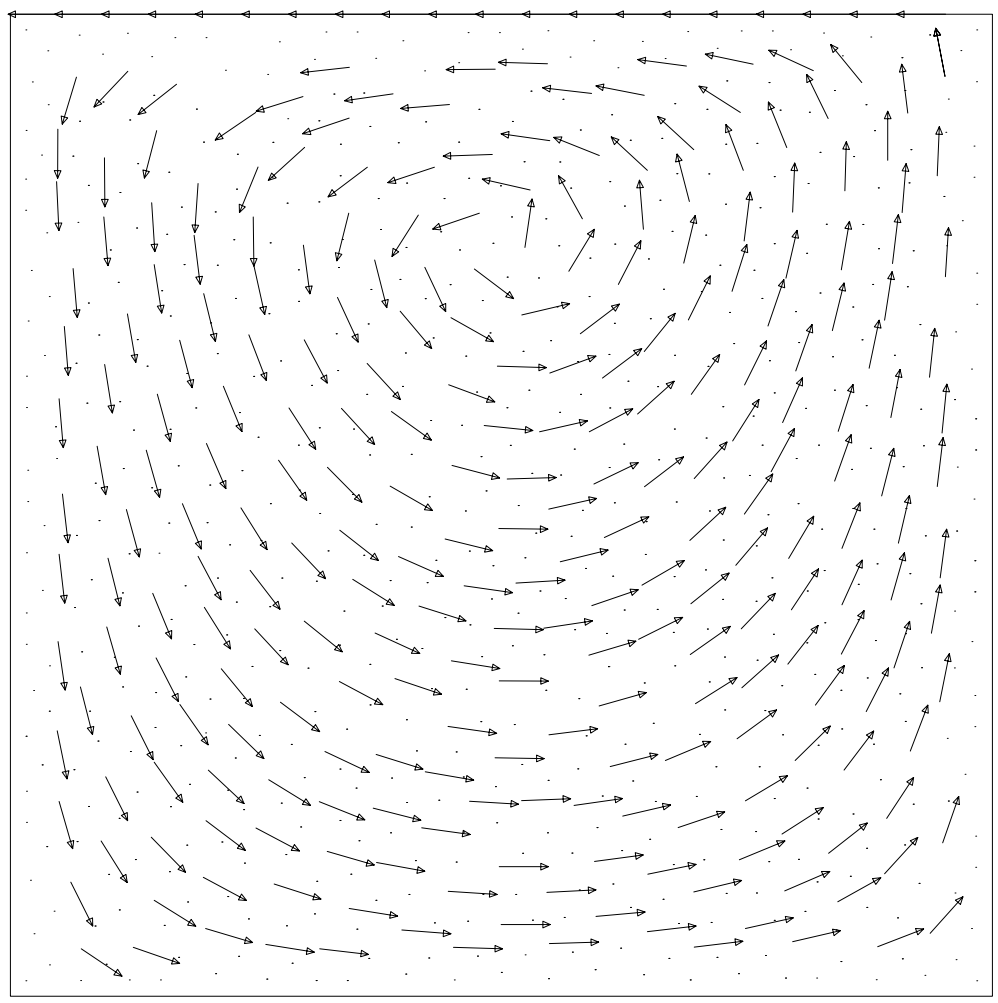

Fig. 2. Velocity field for a steady lid-driving cavity problem.

In order to get accurate incompressible solutions, we use a stream function formulation and solve the resulting biharmonic equation with a pseudospectral Chebyshev technique. The flow kinematics is depicted in Fig. 2.

Now we consider the streamline passing through the point $P=(0.5,0.99)$ and a relaxation time for the molecular orientation $\tau_{b}=T / 17$ (where $T$ is the period associated with the closed trajectory considered). The steady orientation along the streamline is depicted in Fig. 3, where we represent the orientation tensor by means of an ellipse. The ellipse axes directions correspond to the eigenvectors of the orientation tensor and the half of the length of the axes correspond to the eigenvalues.

Remark. This strategy can be applied in any point of the recirculating flow except in the center of rotation. The center of rotation is a singularity point which introduces high perturbations in Eulerian discretisations [18]. Using the technique proposed in this paper, the center of rotation does not introduce any perturbation in the numerical simulation. Furthermore, we have proved that in some cases the solution in the neighborhood of the center of rotation can be extended with continuity to this point [31].

The advection equation governing the molecular extension is non-linear due to the fact that the relaxation time depends on the molecular extension

$$
\frac{\mathrm{d} \lambda}{\mathrm{d} t}=\lambda \operatorname{Tr}(\underline{\underline{s}} \underline{\underline{D}})-\frac{1}{\tau_{s}}(\lambda-1)
$$

with

$$
\tau_{s}=\tau_{0} \mathrm{e}^{-v(\lambda-1)} .
$$

The solution related to a constant relaxation time $(v=0) \tau_{s}=\tau_{0}=T / 17$ and to the molecular orientation shown in Fig. 3 is depicted in Fig. 4 where the extension magnitude is related to the circle diameter used in 


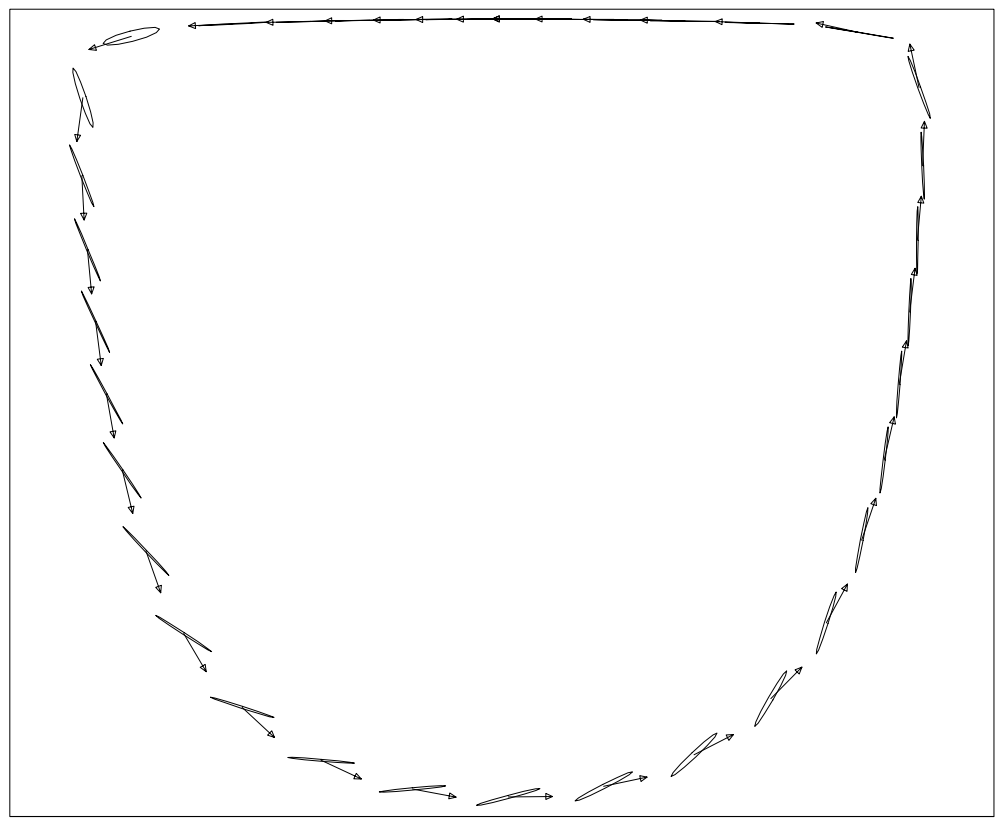

Fig. 3. Steady orientation for $\tau_{b}=T / 10$.

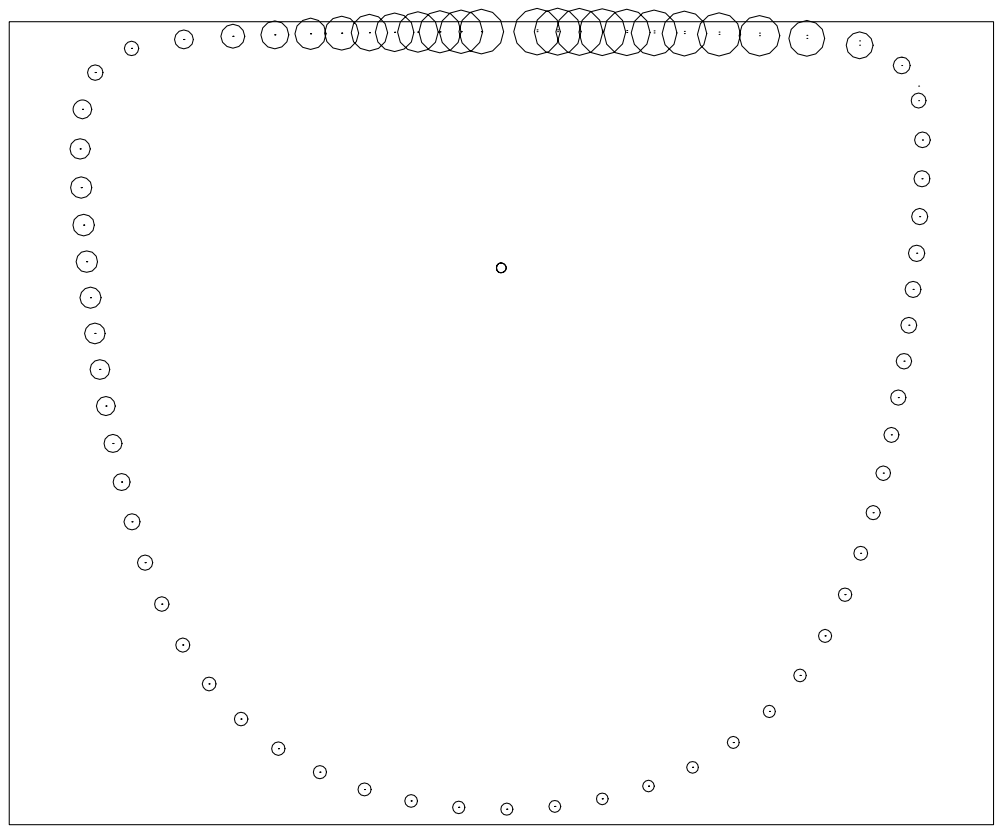

Fig. 4. Steady extension for $\tau_{s}=\tau_{0}=T / 10$.

the representation (a circle associated with the natural molecular extension $\lambda=1$ is depicted at the center of rotation).

In order to solve the non-linear problem $(v \neq 0)$ two strategies have been considered: a fixed point algorithm and a Newton's method. A fourth-order Runge-Kutta scheme with a control step has been used in the integration of the advection equation by the method of characteristics. 


\subsection{A fixed point strategy}

The solution of the non-linear advection problem, Eq. (37), is found with the usual iterative algorithm

$$
\frac{\mathrm{d} \lambda^{n}}{\mathrm{~d} t}=\lambda^{n} \operatorname{Tr}(\underline{\underline{s}} \underline{\underline{D}})-\frac{\mathrm{e}^{v\left(\lambda^{n-1}-1\right)}}{\tau_{0}}\left(\lambda^{n}-1\right),
$$

where $\lambda^{n}$ denotes the molecular extension field at present iteration, $\lambda^{n-1}$ the one associated with the previous iteration, and the fields $\underline{\underline{s}}, \underline{\underline{D}}$ and $\lambda^{n-1}$ only depend on the spatial coordinates. $\lambda^{n-1}$ is the known solution at the previous iteration, and its values can be different all along the closed streamline. The linear problem that results at each iteration is solved by means of the proposed strategy which keeps the solution periodicity. As a first periodic estimation of the molecular extension, needed in order to start the iteration procedure Eq. (39), the natural extension is considered $\left(\lambda^{0}=1\right)$.

\subsection{Newton's method}

Newton's linearization of Eq. (37) results

$$
\frac{\mathrm{d} \Delta \lambda^{n}}{\mathrm{~d} t}-\Delta \lambda^{n} \operatorname{Tr}(\underline{\underline{s}} \underline{\underline{D}})+\Delta \lambda^{n} \frac{v\left(\lambda^{n-1}-1\right)+1}{\tau_{0}} \mathrm{e}^{v\left(\lambda^{n-1}-1\right)}=-R\left(\lambda^{n-1}\right),
$$

where $\Delta \lambda^{n}$ is the correction to apply to the field $\lambda^{n-1}$, and $R\left(\lambda^{n-1}\right)$ the residual at the previous iteration defined by

$$
R\left(\lambda^{n-1}\right)=\frac{\mathrm{d} \lambda^{n-1}}{\mathrm{~d} t}-\lambda^{n-1} \operatorname{Tr}(\underline{\underline{s}} \underline{\underline{D}})+\frac{\mathrm{e}^{v\left(\lambda^{n-1}-1\right)}}{\tau_{0}}\left(\lambda^{n-1}-1\right) .
$$

We consider again $\lambda^{0}=1$ as the initial extension in numerical examples, solving the linear equation related to the correction equation (40) at each iteration by means of the proposed technique in order to keep the periodicity of the solution corrections.

\subsection{Numerical results}

From both strategies results the same solution, however we cannot conclude about its unicity. The nonlinear solution related to Eqs. (37) and (38) with $v=0.9$ and a $\tau_{0}$ value equal to $T / 17$ is shown in Fig. 5. As the relaxation time decreases with the molecular extension, the final molecular extension is lower than the one associated to the linear case.

Fig. 6 shows the evolution of the $L^{2}$-norm of the residual $\left(\left\|R\left(\lambda^{n}\right)\right\|_{L^{2}}\right)$ in each iteration for both procedures: the fixed point and Newton's strategy. In both cases the residual is defined as the difference between two consecutive solutions in a point on the closed trajectory. In our case the starting point for numerical integrations $P=(0.5,0.99)$ has also been chosen for the residual evaluation.

In the numerical example considered here, at the first iteration of the fixed point algorithm, we find that the solution of the homogeneous equation associated with Eq. (39), after a period, $\lambda_{h}(T)$, is very close to zero

$$
\lambda_{h}(T) \approx 0 .
$$

Thus, according with the procedure described in Section 3.1, the periodicity condition for the molecular extension $\lambda$ results

$$
\alpha \lambda_{h}(T)+\lambda_{c}(T)=\alpha+1,
$$

where $\lambda_{c}(T)$ is the particular solution of Eq. (39) related to the initial condition $\lambda_{c}(t=0)=1$. So, the parameter $\alpha$ results

$$
\alpha \approx \lambda_{c}(T)-1
$$




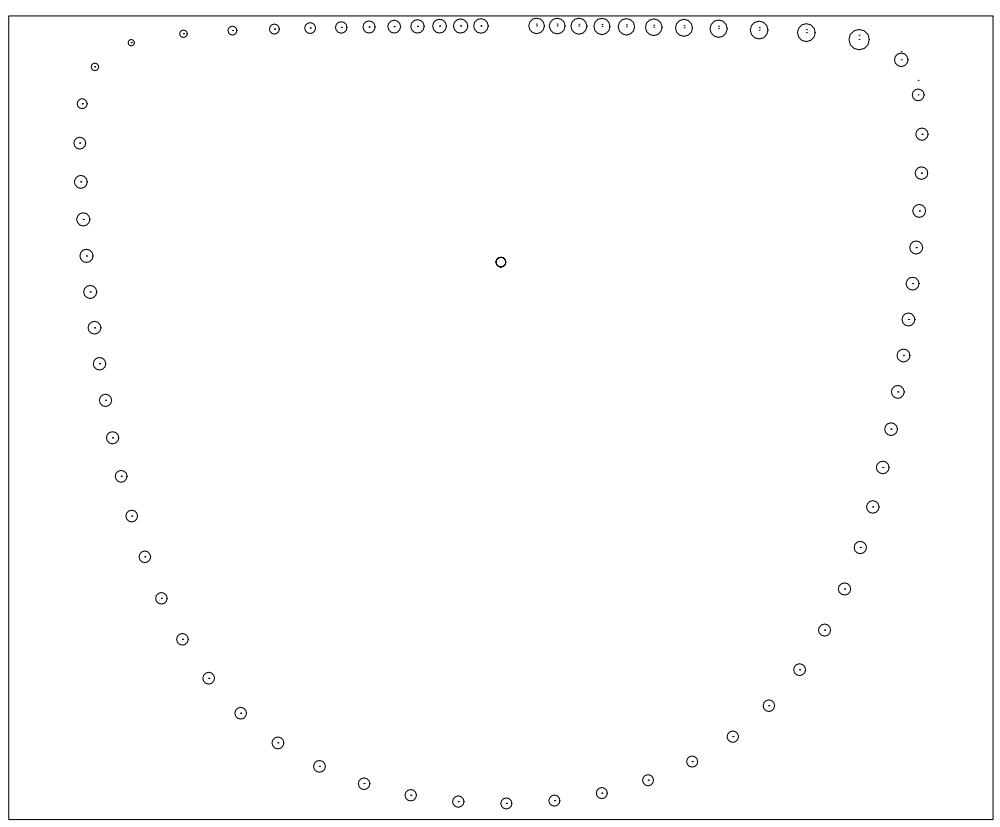

Fig. 5. Non-linear solution for molecular extension.

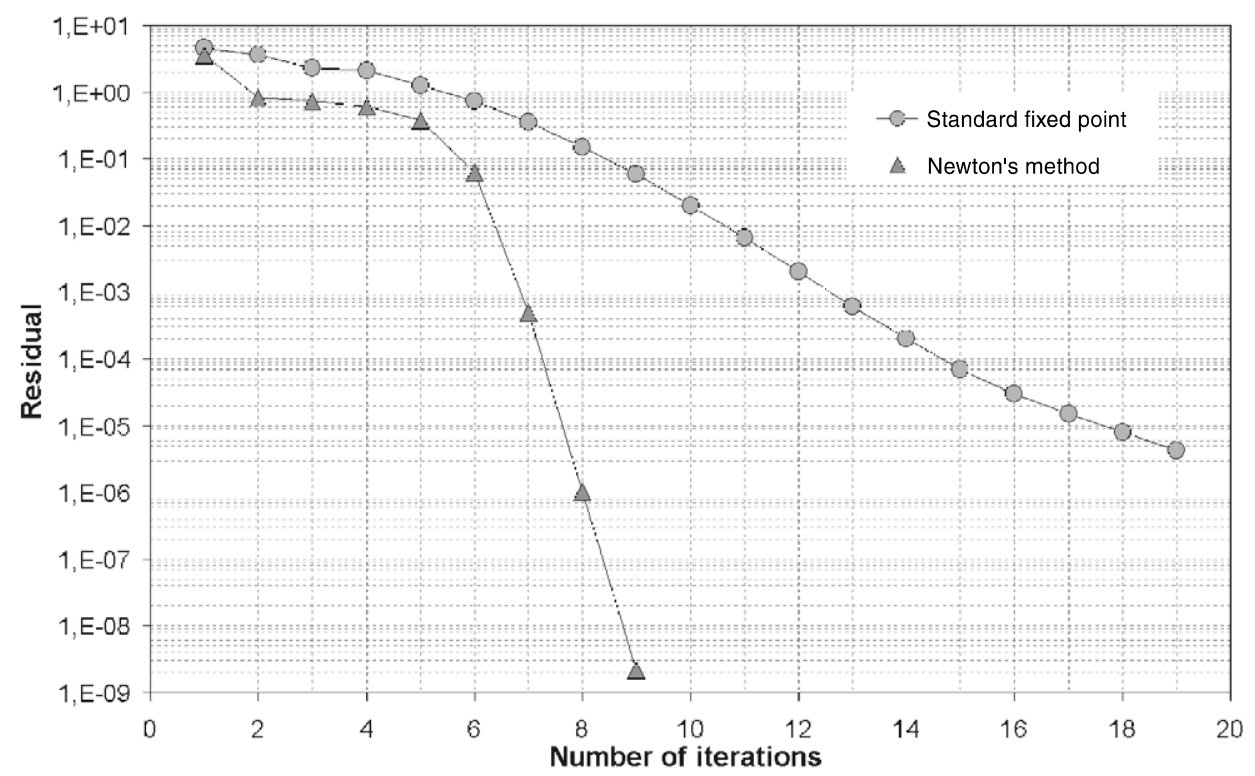

Fig. 6. Residual evolution in logarithmic scale.

and the initial value of $\lambda$ to impose at the point $P$ at this iteration, in order to obtain a periodic solution for Eq. (39), will be given by $1+\alpha \approx \lambda_{c}(T)$. Effectively, in this case we can verify that $\lambda_{c}(T)$ does not depend on the particular initial value considered in the integration of Eq. (39).

This fact induces us to improve the fixed point strategy in the following way. We start from $\lambda^{0}=1$ (at each point on the closed trajectory), and we solve the linear equation applying the method of characteristics

$$
\frac{\mathrm{d} \lambda^{1}}{\mathrm{~d} t}=\lambda^{1} \operatorname{Tr}(\underline{\underline{s}} \underline{\underline{D}})-\frac{\mathrm{e}^{v\left(\lambda^{0}-1\right)}}{\tau_{0}}\left(\lambda^{1}-1\right)
$$




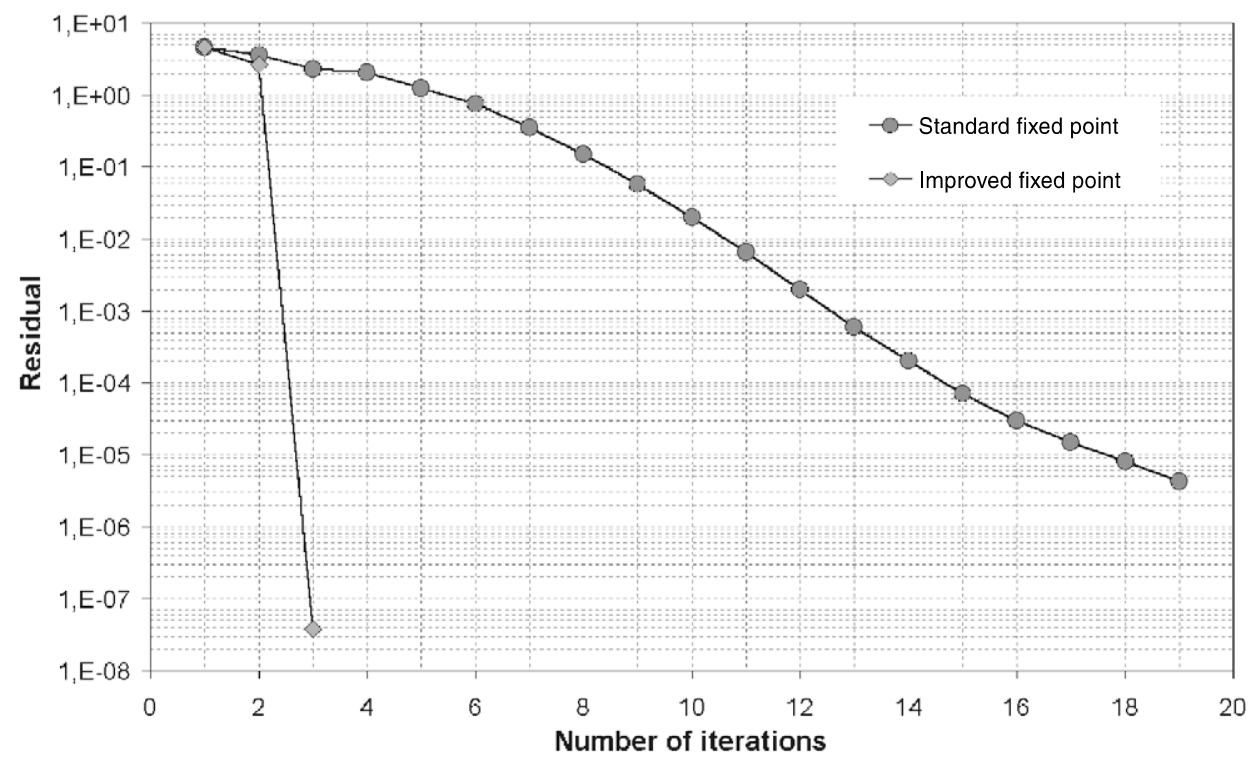

Fig. 7. Residual evolution in logarithmic scale.

to obtain the periodic solution $\lambda^{1}$. We note by $\lambda_{0}^{1}$ the solution $\lambda^{1}$ at the point $P$. Now, we can integrate, by the method of characteristics once more, Eq. (37)

$$
\frac{\mathrm{d} \lambda^{*}}{\mathrm{~d} t}=\lambda^{*} \operatorname{Tr}(\underline{\underline{s}} \underline{\underline{D}})-\frac{\mathrm{e}^{v\left(\lambda^{*}-1\right)}}{\tau_{0}}\left(\lambda^{*}-1\right)
$$

from the initial value

$$
\lambda^{*}(t=0)=\lambda_{0}^{1} .
$$

The periodic solution at the next iteration of the fixed point algorithm, $\lambda^{2}$, is found integrating again the linear advection equation given by Eq. (39) with the non-linear term evaluated from the non-periodic intermediate solution $\lambda^{*}$, i.e.

$$
\frac{\mathrm{d} \lambda^{2}}{\mathrm{~d} t}=\lambda^{2} \operatorname{Tr}(\underline{\underline{s}} \underline{\underline{D}})-\frac{\mathrm{e}^{v\left(\lambda^{*}-1\right)}}{\tau_{0}}\left(\lambda^{2}-1\right)
$$

This process must continue until convergence. This version of the fixed point algorithm will be called improved fixed point algorithm.

Fig. 7 compares the evolution of the $L^{2}$-norm of the residual $\left(\left\|R\left(\lambda^{n}\right)\right\|_{L^{2}}\right)$ for the standard and the improved fixed point algorithms.

The numerical procedure proposed in this paper seems to suit to the treatment of non-linear advection problems better than the Lagrangian strategy discussed in Section 2, mainly when the advection equation is tensorial. In this case the strategy described in Section 2 requires a correction at each iteration for all the components of the initial estimation. The associated tangent matrix is generally evaluated numerically and problems on the solution accuracy can appear in some cases [27].

\section{Conclusions}

In this paper we present a strategy to solve steady non-linear advection problems defined in steady recirculating flows. This numerical strategy gives a solution of the non-linear advection problem, verifying 
exactly the periodicity condition along the closed streamlines. In consequence, it allows to compute an accurate steady solution of the problem.

Furthermore, as in the numerical iteration, the method of characteristics is used, the accuracy of the solution can be improved refining the time step used in the integration. This technique combines a standard treatment of the non-linearity by means of a fixed point or a Newton's scheme, with a more original strategy to solve the associated linear problems which keeps the required solution periodicity along the closed trajectories and avoid the use of periodic Eulerian interpolations and the solution of the associated linear systems.

A comparison between our strategy and other techniques in a steady recirculating flows, for PomPom, Oldroyd B, and other viscoelastic models, is a further work that has to be done. As the solution of the non-linear advection equation obtained with our technique verifies the stationarity in each point of the flow domain, it can be used to evaluate the solution obtained with other Eulerian discretization techniques.

\section{Acknowledgements}

We acknowledge the "Société Rhéo" branch of "Thermo Haake GMBH, Germany" for providing together with the "Association Nationale pour la Recherche Technologique (ANRT) - France", a research position for one of us (G. Chaidron).

\section{References}

[1] G.L. Hand, A theory of anisotropic fluids, J. Fluid Mech. 13 (1962) 33-46.

[2] G.K. Batchelor, Slender-body theory for particles of arbitrary cross-section in Stokes flow, J. Fluid Mech. 44 (1970) $419-440$.

[3] G.K. Batchelor, The stress generated in non-dilute suspensions in elongated particles by pure straining motion, J. Fluid Mech. 46 (1971) 813.

[4] E.J. Hinch, L.G. Leal, Constitutive equations in suspension mechanics. Part I, J. Fluid Mech. 71 (1975) 481-495.

[5] E.J. Hinch, L.G. Leal, Constitutive equations in suspension mechanics. Part II, J. Fluid Mech. 76 (1976) $187-208$.

[6] S.M. Dinh, R.C. Armstrong, A rheological equation of state for semi-concentrated fiber suspensions, J. Rheol. 28 (1984) 207.

[7] Ch.L. Tucker III, Flow regime for fiber suspensions in narrow gaps, J. Non-Newtonian Fluid Mech. 39 (1991) $239-268$.

[8] F. Meslin, Propriétés rhéologiques des composites fibres courtes á l'etat fondu, Ph.D. Thesis, Ecole Normale Superieure de Cachan, 1997.

[9] R. Keunings, Viscoelastic flows, in: S.G. Advani (Ed.), Flow and Rheology in Polymer Composites Manufacturing, Elsevier, Amsterdam, 1994.

[10] F.P.T. Baaijens, Experimental and Numerical Analysis of Viscoelastic Flows, PPS-15, Polymer Processing Society, Netherlands, 1999.

[11] T.C.B. McLeish, R.G. Larson, Molecular constitutive equations for a class of branched polymers: the Pom-Pom polymer, J. Rheol. 42 (1) (1998) 81-110.

[12] W.M.H. Verbeeten, G.W.H. Peters, F.P.T. Baaijens, Differential constitutive equations for polymer melts: the extended Pom-Pom model, J. Rheol. 45 (4) (2000) 823-843.

[13] C. Givler, M.J. Crochet, R.B. Pipes, Numerically predicted fiber orientation in dilute suspensions, NUMIFORM, Pineridge, Swansea, 1982, pp. 559-575.

[14] J. Rosenberg, M. Den, R. Keunings, Simulation of non-recirculating flows of dilute fiber suspensions, J. Non-Newtonian Fluid Mech. 37 (1990) 317-345.

[15] G. Ausias, Etude de l'extrusion de tubes en polymères thermoplastiques chargés de fibres courtes, Ph.D. Thesis, E.N.S.M.P, 1991.

[16] M.C. Altan, S.I. Guçeri, R.B. Pipes, Anisotropic channel flow of fiber suspensions, J. Non-Newtonian Fluid Mech. 42 (1992) 6583.

[17] K. Chiba, K. Nakamura, Numerical solution of fiber suspensions flow through a complex channel, J. Non-Newtonian Fluid Mech. 78 (1998) 167-185.

[18] A. Poitou, F. Chinesta, R. Torres, Numerical simulation of the steady recirculating flows of fibers suspensions, J. Non-Newtonian Fluid Mech. 90 (2000) 65-80.

[19] H. Henry de Frahan, V. Verleye, F. Dupret, M.J. Crochet, Numerical prediction of fiber orientation in injection molding, Polymer Engrg. Sci. 32 (4) (1992) 254-260.

[20] S.T. Chung, T.H. Kwon, Numerical simulation of fiber orientation in injection molding of short-fiber-reinforced thermoplastics, Polymer Eng. Sci. 35 (7) (1995) 604-618.

[21] J. Azaiez, R. Guénette, A. Ait-Kadi, Investigation of the abrupt contraction flow of fiber suspensions in polymeric fluids, J. NonNewtonian Fluid Mech. 73 (1997) 289-316. 
[22] F. Chinesta, A. Poitou, R. Torres, Numerical prediction of the fiber orientation in steady flows, Revue Européenne des Eléments Finis 8 (4) (1999) 355-374.

[23] B. Souloumiac, Etude rhéologique, modélisation et simulation numérique de l'ecoulement des thermoplastiques chargés de fibres courtes, Ph.D. Thesis, Ecole National Supérieure des Mines de Paris, 1996.

[24] R.E. Evans, K. Walters, Further remarks on the lip-vortex mechanism of vortex enhancement in planar-contraction flows, J. NonNewtonian Fluid Mech. 32 (1989) 95-105.

[25] P. Townsend, K. Walters, Expansion flows of non-Newtonians liquids, Chem. Engrg. Sci. 49 (5) (1993) $749-763$.

[26] F. Chinesta, G. Chaidron, On the steady solution of linear advection problems in steady recirculating flows, J. Non-Newtonian Fluid Mech. 98 (2001) 65-80.

[27] F. Chinesta, F. Olmos, A. Poitou, R. Torres, Orientación de las fibras en flujos recirculantes, Asociación Española de Materiales Compuestos, Seville, 1995, pp. 395-402.

[28] G. Chaidron, F. Chinesta, Further considerations on steady recirculating flows involving non-Newtonian fluids, J. NonNewtonian Fluid Mech. (submitted).

[29] A. Poitou, F. Chinesta, F. Olmos, R. Torres, Numerical simulation of reinforced thermoplastic flows, in: Finite Elements in Fluids, CIMNI-Pineridge Press, Swansea, 1993, pp. 1178-1187.

[30] O. Pironneau, Finite Element Method for Fluids, Wiley, New York, 1989.

[31] G. Chaidron, F. Chinesta, On the solution of advection equation in the vicinity of the singularities for steady recirculating flows, in: Proceedings of the 4th International ESAFORM Conference on Material Forming, Liège, Belgium, vol. 1, 2001, pp. 19-22. 